\title{
JETS FROM ACCRETING WHITE DWARFS
}

\author{
J. L. Sokoloski, ${ }^{1,2}$ S. J. Kenyon, ${ }^{1}$ C. Brocksopp ${ }^{3}$ C. R. Kaiser, ${ }^{4}$ and E. M. Kellogg ${ }^{1}$
}

\section{RESUMEN}

Los flujos colimados procedentes de los discos de acreción de las enanas blancas juegan un papel nuny importante al estudiar los "jets" en astrofísica.

\section{ABSTRACT}

Collimated outflows from accreting white dwarfs have a vital role to play in the study of astrophysical jets.

\begin{abstract}
Key Words: ACCRETION, ACCRETION DISKS - BINARIES: SYMBIOTIC - STARS: WINDS, OUTFLOWS - WHITE DWARFS
\end{abstract}

Observationally, jets are associated with systems where material is accreted though a disk. Theoretically, accretion disks provide the foundation for many jet models. Perhaps the best-understood of all accretion disks are those in cataclysmic variable stars (CVs). Since the disks in other accreting whitedwarf (WD) binaries are probably similar to $\mathrm{CV}$ disks (at least to the extent that one does not expect complications such as advection-dominated flows, for example), with WD accretors we have the advantage of a relatively good understanding of the region from which the outflows probably originate.

There are three main classes of accreting WDs: CVs, supersoft X-ray binaries (SSXBs), and symbiotic stars (see Table 1). In terms of binary separation and orbital period, they form a hierarchy: CVs are the most compact, with orbital periods of the order of hours; SSXBs are slightly wider, with orbital periods from hours to days; and symbiotics are the widest, with orbital periods on the order of years. The WD luminosities do not form such a simple hierarchy - in CVs the luminosity of the accreting WD is low, but in both SSXBs and symbiotics it is high enough that it would be difficult to produce it by accretion alone. Thus, some of the accreted fuel in both SSXBs and most symbiotics probably undergoes thermonuclear fusion burning in a shell on the WD surface. To produce quasi-steady shell burning, either a time-averaged accretion rate of roughly $10^{-7}$ to $10^{-8} \mathrm{M}_{\odot} / \mathrm{yr}$ or a recent 'thermal pulse' of runaway nuclear shell burning may be required. High accretion-rate CVs show modestly collimated disk winds, but they have not been observed to produce

\footnotetext{
${ }^{1}$ Harvard-Smithsonian CfA, 60 Garden St., Cambridge, MA 02138, USA.

${ }^{2}$ NSF Astronomy \& Astrophysics postdoctoral fellow.

${ }^{3}$ Mullard Space Science Laboratory, LCL, Surrey, UK

${ }^{4} \mathrm{U}$. Southampton, Hampshire SO17 1BJ, UK.
}

TABLE 1

ACCRETING WHITE IDWARFS

\begin{tabular}{rccc}
\hline & $\begin{array}{c}\text { Cataclymic } \\
\text { Variables }\end{array}$ & $\begin{array}{c}\text { Supersoft } \\
\text { Soures }\end{array}$ & $\begin{array}{c}\text { Symbiotic } \\
\text { stars }\end{array}$ \\
\cline { 2 - 3 } Size: & Snall & Medium & Large \\
Donor: & Dwarl & Evolved & Giant \\
$L_{W D^{2}:}$ & Few & $10^{4}$ & $10^{3}$ \\
$M I:$ & Low & High & High \\
$M$ Mech: & Stable & Unstable & Wind \\
& RLO & RLO & \\
Jets? & NO & YES & YES \\
\hline
\end{tabular}

${ }^{a}$ Units of $L_{\%} . \quad{ }^{b} \mathrm{RLO}$ is short for Roche-lobe overflow.

narrow, collimated jets. Both SSXBs and symbiotics, however, have. Based on this meeting, hot central stars of planetary nebulae ( $\mathrm{PNe}$ ) make an appropriate fourth column in Table 1 . Some PAe also produce collimated bi-polar outflows.

Symbiotic stars are numerous, unambiguous accreting systems, and close enough for their collimated outflows to be spatially resolved. We therefore concentrate on symbiotics. The IVD in a symbiotic is fed from the wind of the red giant. This wind forms a nebula that is partially ionized by radiation from the hot WD. Shock heating of nebular material by collimated ejecta could help make symbiotic-star jets observable. Collimated outflows from srmbiotics are often transient. and some are associated with poorly-understood classical symbiotic outbursts.

Symbiotic jets range in size from tens of milliaresec to tens of archece. (orresponding to physical sizes: of tens to thousands of AU. Inferred flow speteds are hundreds to thousands of $\mathrm{km} / \mathrm{s}$ (although cridence for relativist ic electrons has also been found: Crocker et al. 2001, Taylor et al. 1989). Evidenct for collimated outflows has been found in practically every type of symbiotic star t.g. in systems with and 


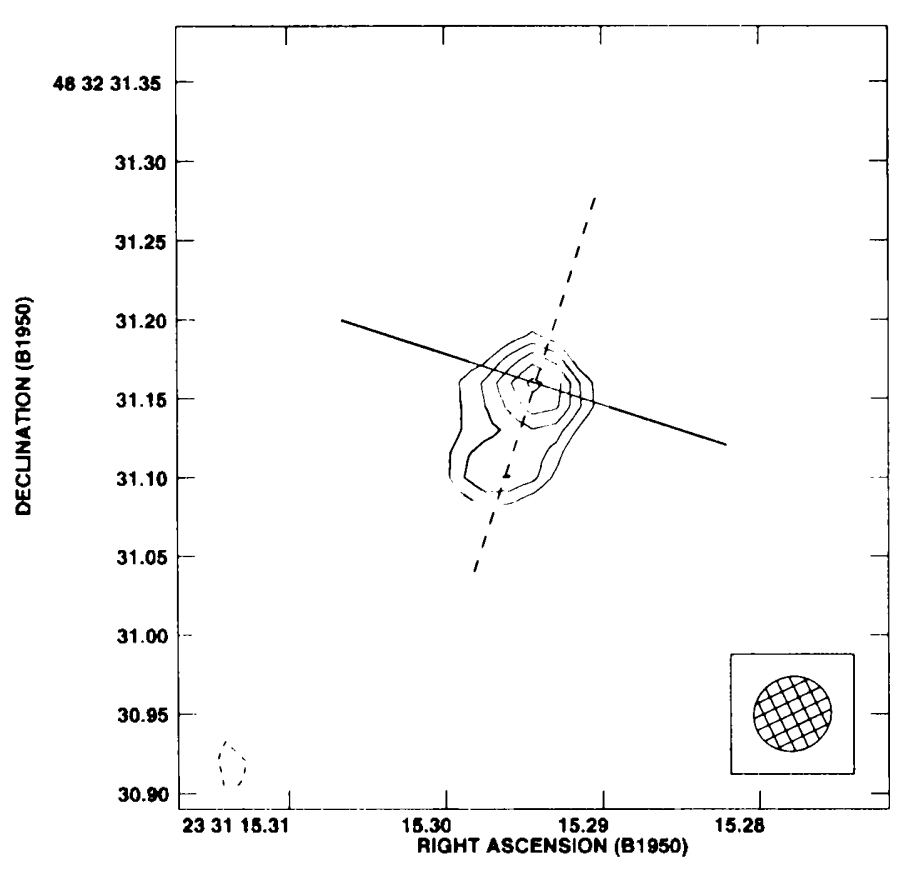

Fig. 1. Transient extended radio structure in $\mathrm{Z}$ And. The line is the projected perpendicular to the orbital plane.

without nuclear shell burning on the WD, in those with strong and weak WD magnetic fields, in 'Dtype' and 'S-type' systems with Mira/non-Mira mass donors, in symbiotic recurrent novae and systems with classical symbiotic outbursts. At least ten of the roughly 200 known symbiotics have shown either spectroscopic or imaging (radio or optical) evidence for collimated outflows. However, given their transient nature and small angular extent on the sky, it is likely that many symbiotic jet ejections have gone undetected, and that the fraction of symbiotics that produce jets is much higher that the currently known $5 \%$. We discuss threc examples below.

To demonstrate the observational challenges faced in the study of WD jets, we review $Z$ And. The prototypical symbiotic star, Z And has a strong WD magnetic field (Sokoloski \& Bildsten 1999), has a high WD luminosity, and experiences classical symbiotic outbursts. Brocksōp et al. (2004) monitored $\mathrm{Z}$ And in the radio during the large outburst beginning in 2000. One year after the start of the optical outburst, a small (60 mas) but significant extended radio structure appeared (see Figure 1). Within one month, the extended structure had faded. Because it was elongated perpendicular to the orbital plane (see Schmid and Schild 1997), they interpreted the transient structure as a jet-like ejection. The outflow velocity was at least $400 \mathrm{~km} / \mathrm{s}$, and the radio flux density indicated that the jet emission was likely to have been thermal Bremsstrahlung.

Our second example, $\mathrm{CH}$ Cyg, highlights the link between WD accretors and other types of jet- producing systems. CH Cyg has a low enough WD luminosity that it is unlikely to contain nuclear shell burning, and it has large-amplitude CV-like optical flickering, presumably from an accretion disk. It moves between optical brightness states rather than showing well-separated outbursts, and it tends to produce jets as it changes between these states. On one of the occasions of radio-jet ejection (Karovska et al 1998), Sokoloski \& Kenyon (2003) found that the most rapid flickering disappeared. If the fastest variations come from the region closest to the WD an idea supported by eclipse-mapping studies in CVs and the theoretical expectation that variability time scale is related to viscous and/or dynamical time scales, both of which are shorter for smaller disk radii - then it appears that the inner disk was disrupted, or emission from the inner-disk region suppressed when the jet was produced. A similar relationship between jet ejection and an change in the inner disk has been found in some X-ray binaries.

Our final example, R Aqr, is the only WD currently known to have an X-ray jet. The X-ray emission from this nearby, Mira-containing, long-period symbiotic was examined by Kellogg et al. (2001). At energies above $1 \mathrm{keV}$, only the central point source was detected. At energies below $1 \mathrm{keV}$, they saw bipolar structure with an X-ray spectrum indicative of shock-heated plasma out of thermal equilibrium. Although some $\mathrm{X}$-ray features corresponded to features in the radio map, the main $\mathrm{X}$-ray bright spot in the northern jet was further from the central object than the main radio bright spot. Follow-up Chandra and VLA observations performed 3 years later, in 2003, show significant changes in the jet structure in both the X-ray and radio images (Kellogg et al. 2004).

Finding jets from WDs is a challenge. Nonetheless, two out of three classes of accreting WDs have shown evidence for jets (three out of four if PNe are included). The diversity of jet-producing WD systems indicates that in some sense jets must be easy to produce. One element that could be common to all jet-producing WDs is an accretion disk, perhaps with accretion rate or size above some threshold level.

\section{REFERENCES}

Brocksopp, C., et al. 2004, MNRAS, 347, 430

Crocker, M. et al. 2001, MNRAS, 326, 781

Karovska, M., et al. 1998, JAAVSO, 26, 97

Kellogg, E. M.. et al. 2001, ApJ, 563, L151

Kellogg, E. M., et al. 2004, in preparation

Schmid, H. M. \& Schild, H. 1997, A\&A, 327, 219

Sokoloski, J. L., \& Bildsten, L. 1999, ApJ, 517, 919

Sokoloski. J. L., \& Kenyon, S. J. 2003, ApJ, 584, 1021

Taylor, A. R., Davis, R. J., Porcas, R. W., \& Bode, M. F. 1989, MINRAS, 237,81 